\title{
EXPERIENCE IN THE BROADENING OF A SINGLE-PURPOSE SIMULATION MODEL
}

\author{
Reid Kress \\ Pete Bereolos \\ Karen Bills \\ James Clinton \\ Jack Dixon \\ Phil Dunn \\ Julie Moore \\ Rob Wilson \\ Y-12 National Security Complex \\ Bear Creek Road \\ Oak Ridge, Tennessee 37831
}

\begin{abstract}
Simulation models are often developed for a single purpose. However, once a model is accepted by management and other stake-holders, it is quite common and desirable to wish to broaden the application of the model to several areas. This is not always a straight-forward evolution because a model designed to evaluate one performance measure may not be well-suited for others. This paper summarizes the experience of the Y-12 National Security Complex's simulation modeling group in broadening its equipment-scoping simulation model into a model that could examine plant mass balance, model internal products including complex feedback loops, include chemical sampling and analysis, evaluate in-process storage, and perform basic scheduling analyses. Results of the effort were successful and the paper concludes that single-purposemodel broadening can be achieved with the correct mix of planning and execution.
\end{abstract}

\section{INTRODUCTION}

Models developed for use in designing new processes, modifying existing processes, optimizing existing processes, setting production goals, etc. are often built in separate phases in response to customer needs and requests. While this is a natural consequence of modeler/customer interaction, it is not, however, most conducive to the production of a robust, long-lived, model. Often, models are built and then modified, extended, redone, re-used, re-directed until the end product is not only no longer recognizable from the initial model but it is also often difficult to use and almost impossible to maintain.
This paper discusses lessons learned in Y-12's transitioning of one of its substantial models from a basic equipment-only model that was used to support conceptual design of a planned new facility to a larger, moresophisticated model that has broader capabilities and added functionality that is now being used to support preliminary design. This paper is divided into the following basic discussion topics:

- Introduction to the goals of Y-12's planned Uranium Processing Facility (UPF) and its associated modeling effort

- $\quad$ Added Basic Functionality to the UPF Model

- Mass Balance

- Internal Products

- Analytical Chemistry

- Lean-Manufacturing Related Additions

- In-Process Storage

- Scheduling

- Number of Personnel

- Miscellaneous Model and Administrative Changes

- Model modifications to address continued block growth

- Configuration and Infrastructure Management with a Large Team

- Managing Many Long-Term Runs 


\section{GOALS OF THE UPF MODELING EFFORT}

The Y-12 National Security Complex is a premier manufacturing facility dedicated to making our nation and the world a safer place. Operated by B\&W Y-12 for the National Nuclear Security Administration, Y-12 plays a vital role in the Department of Energy's Nuclear Weapons Complex. Y-12 helps ensure a safe and reliable U.S. nuclear weapons deterrent, retrieves and stores nuclear materials, fuels the nation's naval reactors, and performs work for other government and private-sector entities. As a consequence of this mission, Y-12 makes dozens of products, having hundreds of parts, each with many different process steps associated with manufacturing components, building sub-assemblies, or assembling a final product. In addition, Y-12 disassembles weapons in order to support stockpile reduction efforts and to retrieve high-value materials and components. Y-12's technology-based missions can be distributed into the following broad categories:

- Production and rework of complex nuclear weapon components and secondaries;

- Receipt, storage, and protection of special nuclear materials;

- Quality evaluation and enhanced surveillance of the nation's nuclear stockpile;

- Dismantlement of weapon secondaries and disposition of weapon components;

- Prevention of the spread of weapons of mass destruction; and

- Support work for DOE and other federal agencies.

The Y-12 National Security Complex is undergoing significant modernization in an effort to reduce the facility size and the costs associated with operating and maintaining a large infrastructure. As part of this modernization,
Y-12 is attempting to consolidate operations from many separate locations within the plant to a single large manufacturing facility named the Uranium Processing Facility (UPF). One of the primary goals of this consolidation is the streamlining of the manufacturing processes at $\mathrm{Y}-12$ and a concomitant reduction of required manufacturing infrastructure. To determine what numbers and types of machines are needed in the UPF to accommodate future schedules in support of DOE missions, the Y-12 simulation modeling team has developed several large models that are described in Kress et al. (2007 and 2006). The first reference describes a supply chain model which is not the subject of this paper and the other reference describes a discrete-event model programmed in Extend ${ }^{\mathrm{TM}}$, a discreteevent simulation package that is developed and sold by Imagine That Inc. <http: //www. extendsim.com/>. The discrete event model is quite large, containing over 100,000 blocks and requiring almost $200 \mathrm{MB}$ of disk space to store and is based upon a job-shop concept. An example front screen of a simplified job-shop model is shown in Figure 1.

The model was originally developed and used for the specific purpose of determining the amounts required for each of the over one-hundred different pieces of equipment needed in the UPF. The model was developed by a team of three engineers working with numerous subject matter experts and was subsequently used over the period of a year and a half to evaluate the proposed equipment needs. Equipment numbers were incorporated into a conceptual design document and used to help secure funding for preliminary design. To support preliminary design, the discrete event model was "dusted off" and modifications were begun to broaden the scope of the model. Designers wanted to include mass balance on all key products, internally generated products (e.g. waste streams, rework, and samples), analytical chemistry laboratory, and in-process storage. 


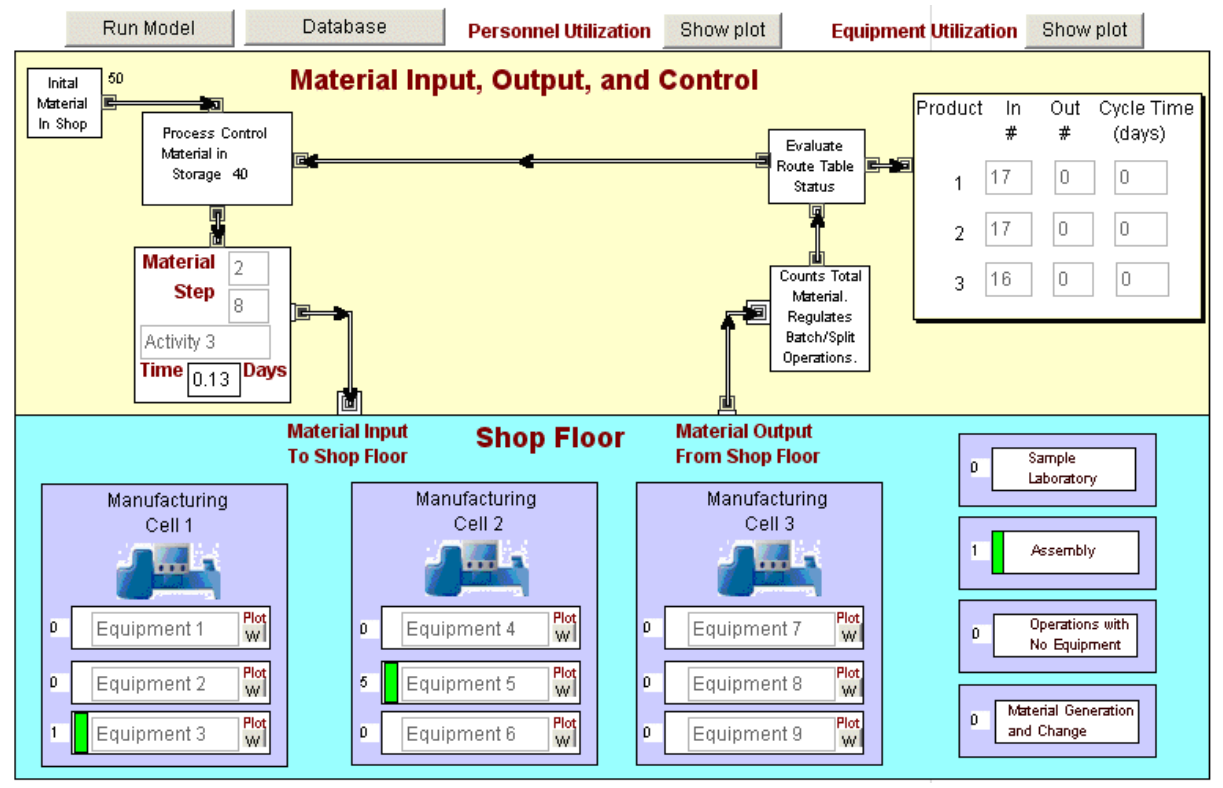

Figure 1: Front page of a simplified version of a job-shop discrete event model. This example contains only three pieces of equipment rather than the over one hundred in the actual UPF model.

This new scope represented a significant set of modifications to the existing model and many very useful lessons were learned in the process. Along with the added scope, the model level of detail also increased (but not to the same extent as the scope broadened).

This paper looks at each of the new scope modifications as well as a few related topics and provides guidance to other groups faced with similar modeling requests. Note that these "scope broadening" requests are not uncommon. Quite often a model is designed and successfully used for a single purpose, the customer gains confidence and sees the utility of a simulation model, and, as a consequence of the good first effort understands more of his/her issues and wishes to expand the modeling effort; often in many directions. Thus, this is a natural consequence of good modeling; however, the resulting model is more complex, Chwif et al. (2000), and has an associated set of new requirements some of which are discussed herein.

\section{THE EXPANDING MODEL}

The growth of the UPF simulation model over time is shown in Figure 2. The growth figure is characterized by three distinct periods: an initial development period associated with adding features to the base model during the early conceptual design period ( $\sim 0$ to $\sim 9$ months), followed by a period of no growth where the model was used hundreds of times for different analyses, and ending in another period of development to include new features to accommodate questions associated with preliminary design. Currently, model functionality is directly related to size. Add- ing capability directly increases the model size, primarily as a result of the added code. Note that adding processors, resources, or other items does not appreciably increase the model size since these entities are established and accounted for in the model database. However, adding new calculations, logic to model a different process, or logic to disposition a previously un-modeled waste stream does increase the model size. In the future, we desire to invert the relationship and have functionality increase as size decreases by doing the following. 1) Add functionality in one place but do it in such a way that you can reduce functionality in another part of the model (e.g. restructure the database so that machines that could potentially have a large variability in the number required all use one type of generic equipment block that supports this variability and those that have small variability use a different, lessflexible, but smaller generic block). 2) Streamline calculations using built-in functions whenever possible to reduce the total number of blocks. 3) Remove model sections used mainly for debugging and preliminary calculations and save them incase they are needed in the future (this actually reduces overall functionality unless one ties some of the data logging/data-tracking functions to the database and

eliminates block-intensive information and read/write code). 4) Eliminate redundant code portions (redundant code seems easier to produce when one uses a graphical based software package). 


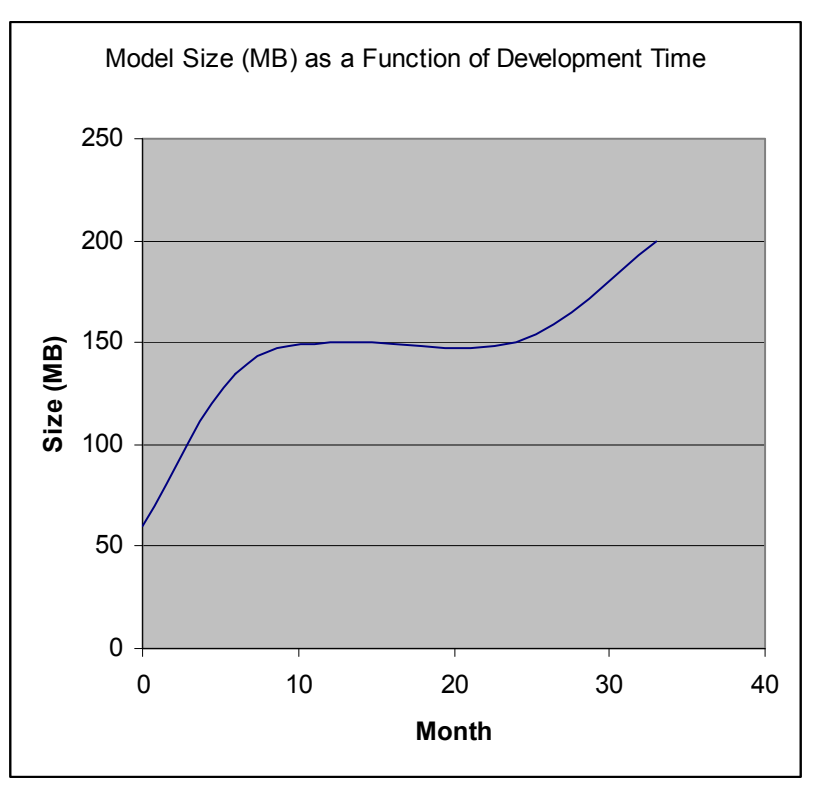

Figure 2: Model size as a function of development time.

\subsection{Adding Basic Functionality to the UPF Model}

After the initial development, application, and analysis stage (at $\sim 22$ months in figure 2), the modeling team was able to identify many future requirements to broaden the model. Each of these requirements necessitated a significant change to the model and its basic concept and are discussed separately below.

\subsubsection{Mass Balance}

One of the most significant additions to the model was the need to perform a mass balance on all of the critical materials, on all machines, for every operation. To perform this balance, the model had to be able to relate products (i.e. material) and routes (i.e. machine and operation). Considering the model contained hundreds of products, hundreds of machines, and hundreds of operations, straight-forward implementation in Extend's database would require searching one rather large table (on the order of 10,000 records) or searching many small tables at every operation. A typical model run for the UPF model involves the analysis of thousands of operations and takes about one hour on a high-end PC. Adding a large database search to every operation would likely increase the execution time by an order of magnitude. This was unacceptable. To alleviate this increase in execution time required the development of an Open Database Connectivity (ODBC) connection to an Access database. The product/route/operation table is established in Access, the search is then executed in Access on each operation, and results returned to Extend. This so- lution worked perfectly, increasing run time by less than a factor of two. The modeling team recognizes that there exists an overhead cost (time) associated with the ODBC calls to and from Access; however, tests show that the "break even" size for using ODBC calls versus a "looping type table lookup written in Extend" was approximately one thousand records. Note, however, that because the ODBC routine required new custom code development, it also necessitated the writing of a new Software Quality Assurance (SQA) plan per Y-12's standard practices. This type of customization has been done in previous software projects, thus the new SQA plan required only two persondays to write, test, and process. Had the team not had significant experience with Y-12's SQA process, this would have been a large burden and could have negated the gains of developing the ODBC connection.

\subsubsection{Internal Products}

Another addition to the model capability was the inclusion of internal products. Internal products are products that are not scheduled as direct plant outputs but rather are by products of scheduled production. For example, chips and turnings from machining, oxides from casting, scrap materials from dismantlement, general waste streams, etc. are all internal products. Because these products are not scheduled, but are generated from the scheduling and processing of primary products, they require careful analysis, design, and coding of added database structures to properly account for their generation and logical progression through the simulation model. The added supporting internal product database structures were integrated into the model while other, less involved modifications, were also being included. This required very careful model version control. In addition, any "new" internal products had to be given all of the important characteristics of an "originally scheduled" product or they would not properly progress through the remaining portions of the model logic.

\subsubsection{Analytical Chemistry}

Another significant broadening of the model was the need to add a simulated analytical chemistry laboratory. In the first versions of the model, analytical chemical analyses (e.g. material assay, composition, purity, etc.) were simply modeled as an operation in a route table with "analytical chemistry" as the machine and the transport, storage, analysis, report, return times all summed into one operation duration. For the preliminary design stage, the modeling team needed to provide more details on analytical chemistry. It was decided not to add analytical chemistry to the manufacturing model, but instead to have the manufacturing model determine and record exactly when a sample was needed and assume that it was ready in a fixed turn- 
around time. The recorded schedule from the manufacturing model was then used to drive a smaller model of the analytical chemistry laboratory to determine if the laboratory could provide the turn-around time assumed in the manufacturing model. Thus, the team had a set of coupled models. The modeling team would run the bigger manufacturing model, assume typical analytical chemistry turnaround times, generate and output a text file containing a schedule of samples (thousands for a typical multi-year simulation), run the smaller analytical chemistry model with the manufacturing model-generated schedule, and verify turn-around times. Iterations included modifying the assumed turn-around times or changing the configuration of the analytical chemistry model to achieve the assumed turn-around time.

\subsection{Lean-Manufacturing Related Additions}

According to the Lean Enterprise Institute <http://www.lean.org>, a lean enterprise is "A business system for organizing and managing product development, operations, suppliers, and customer relations. Business and other organizations use lean principles, practices, and tools to create precise customer value goods and services with higher quality and fewer defects with less human effort, less space, less capital, and less time than the traditional system of mass production." Obviously, even if one applies lean principles, there are lower limits on the amount of human effort, space, capital, and time required for a given production system. Assuming that the necessary tasks for producing an item are defined, that is, assuming the "Route Tables" (types of machines/work stations needed and process operation times) are defined, a plan can be derived through a lean approach. A first goal for lean manufacturing is to arrive at the number of machines needed for a given operation. The production goal, that is the number of items $\left(\mathrm{n}_{\mathrm{I}}\right)$ to be produced (completed) in a given amount of time, $t$, is known as well as an estimate for the mean operation time $\Delta$ t. A first order estimate of the Reliability, Availability, and Maintainability data such as mean time to repair (MTTR) and number of failures per machine, $\mathrm{n}_{\mathrm{F}}$, and other needed times such as maintenance scheduled during normal working hours per machine, $\mathrm{m}_{\mathrm{S}}$, may be included. The formula for number of machines, $\mathrm{Z}$, needed at any operation on any route table is determined by the available time divided by the required time as shown in equation (1):

$$
\mathrm{Zt} /\left\{\left(\mathrm{n}_{\mathrm{I}} \Delta \mathrm{t}\right)+\left[\left(\mathrm{n}_{\mathrm{F}} \mathrm{MTTR}\right)+\mathrm{m}_{\mathrm{S}}\right] \mathrm{Z}\right\}<1
$$

To obtain a better estimate there are additional considerations. Each operation in a route table needs to be examined for real-world application. For example, the time available may need to be reduced if the operation times can not evenly fit into the time available. For example, if an operation requires 1.5 hours and there is a four hour effective shift, then only $2(1.5$ hours $)=3$ hours are practically available and the time available needs to be reduced by 3/4. Another consideration is recycle and/or reject parts. If there is recycle and the operation in question is subject to that, the number of items through that operation needs to be increased. For example, if there is a 1 in 3 recycle, then the number of items through the operation is multiplied by $1.5(\{3$ processed $\} /\{2$ successful $\}$ ). Multiple recycles can be treated similarly. The number of items through an operation must also be increased if there are rejects down stream of the step. For example, if 1 of 3 is rejected, then the operation must process 1.5 times the expected product.

\subsubsection{In-Process Storage}

When a system is fed at the rate which the slowest process (the bottleneck) can process, then the minimum storage needed is the sum of the operation times of the related route tables, $\Delta \mathrm{t}_{\mathrm{i}}$, divided by the bottleneck time, $\mathrm{t}_{\mathrm{B}}$. That is:

Number of Items in Process (i.e. stored) $=\Sigma \Delta t_{\mathrm{i}} / \mathrm{t}_{\mathrm{B}}$

This formula is, of course, ideal. Engineering judgment would most probably dictate more storage as individual operations are considered and as contingency strategies are derived. (Contingency strategies are plans for repositioning personnel when scheduled processes are temporarily inoperative and can involve more inventories, more storage, and even more machines.) In addition, if items are left at some work stations rather than being sent to a "storage" area, the number left on the machines/work station can be subtracted from the needed storage.

As related to the simulation model, in-process storage required tracking of pre-machine queue sizes to determine how many items were waiting at any point in time. Not only was storage related to machines, but machines were also grouped in functional areas. The tracking and grouping necessitated changes to machine blocks within the model and modifications to the database to accommodate process grouping. As a result of the need to make changes in hundreds of machine blocks, a custom library was established for these blocks.

\subsubsection{Scheduling}

A lean schedule is accomplished by feeding the system only at the rate the bottleneck (the constraint) can process. Feeding more will result in creating inventory in front of the bottleneck and will use personnel earlier in the manufacturing process and will consequently idle them later. Feeding less than the bottleneck rate, assuming that the full bottleneck capacity is needed, will not meet the 
production goal. When fed at the rate of the bottleneck operation, all other machine/work station schedules are natural consequences of the feed and do not generally have to be explicitly defined though they are imminently definable (may be written by inspection). In order to accommodate more sophisticated scheduling, changes were made in the model driving structure. Inventory, parts-onorder, parts being manufactured, and rejected parts were all tracked and an ordering policy was implemented to ensure adequate inventory even in the presence of random rejections. Under- or over-production of parts is one issue; scheduling of specific parts for lean operation is another. This level of scheduling has not yet been implemented but is under development.

\subsubsection{Number of Personnel}

The lean number of personnel is simply the total number of work hours required divided by the hours a single person can contribute to the effort. The real number of personnel will be higher, but the lean number is a starting point. The real number, or closer to the real number, will take into account the nature of the individual steps, the ability to train the personnel in more than one job, vacation, anticipated illnesses, attitude, etc. Of course, support staff will need to be added too. Personnel information in the UPF model is included only in the data base as "a number of hours required from a given labor type" since labor does not constrain work at Y-12.

\subsection{Miscellaneous Model and Administrative Changes}

\subsubsection{Model modifications to address continued block growth}

Loading and initialization of the model typically required on the order of 20 minutes. This time prompted a search and removal of redundant blocks (i.e., some basic code optimization). Also, the modeling team re-organized the equipment to make specialized blocks for those systems not needing multiple pieces of equipment and were able to reduce the block count significantly (approximately by a factor of two).

\subsubsection{Configuration and Infrastructure Management with a Large Team}

The modeling team consists of eight people (the author list!) Ad hoc configuration management with a team of this size does not work. The team is using Windchill Project Link from PTC Corporation <http://www.ptc.com/appserver/mkt/produ cts/home.jsp?k=351> to manage the model changes and create a documentation path. Project Link allows a model to be checked out and used or modified while other team members are producing runs. In addition to Project Link, the "small changes" philosophy is being applied. If a change takes more than one to two hours, then it is designed, implemented, and tested off-line in small models or recent versions of the full model and is moved to the "current" model all at once. No one is allowed to check out the model for long time periods. Communication among the team members is also important so co-location in the same physical location (new building and dedicated modeling area) are required.

In the beginning stages of model modification, configuration testing and regression testing were done using results from a standard test model. Data from the modified model were compared directly to previous results. As the model grew in complexity, simple changes were vetted through subject matter expert review and comparison to standard model results was used only to verify major revisions.

\subsection{Managing Many Long-Term Runs}

Numerous runs are required because of the large number of different products manufactured at Y-12, because of the number of options being considered, and for proper statistical analysis of the random model. In addition, many of the runs are for a one- to two-decade time frame. Managing many long-term runs required setting up a modeling bullpen with several workstations, obtaining several network licenses for Extend, and setting up the ability to do remote logins on little-used servers for the multi-decade runs that often required ten to twelve hours to complete.

\section{CONCLUSIONS AND RECOMMENDATIONS}

Being able to extend a rather large and entrenched model requires thinking along two disparate paths. One path is a conservative, tried-and-true approach of careful planning and implementation of changes, configuration management of both models and data bases, and tight communication between modeling team members. Juxtaposed with the first path, the second approach requires the development and implementation of out-of-the-box approaches such as the development of an ODBC connection to do rapid data base queries or clever use of data base structures for the implementation of an ordering policy. It is of paramount importance that significant changes that could potentially have a strong impact on the model (e.g. inclusion of internal products) be discussed with the entire team to avoid inadvertently damaging other portions of the model especially sections that are currently being revised. Creating libraries for those custom blocks that appear often in the 
model is also necessary at this stage in the model life cycle. Co-location of the team, creating a modeling bullpen, scheduling frequent team meetings all increase the communication that is vital to success of a simulation modeling effort of this magnitude.

Future enhancements to the Extend software might include a modeling tool that could be used to evolve additional support for this kind of development process as a way to rapidly expand/integrate existing models. It could include a check in/check out capability for configuration management and a modeling canvas for model modifications and additions.

An important future direction for the simulation modeling team is to determine how to enhance a model and still have its size decrease while functionality increases. Several steps were suggested at the end of section 3; however, implementing these steps will have diminishing returns as the model is improved. Consequently, significant future reduction in model size will most likely require a heavier emphasis on custom code development.

\section{REFERENCES}

Chwif, Leonardo, Barretto, Marcos, and Paul, Ray, On Simulation Model Complexity, In Proceedings of the 2000 Winter Simulation Conference, eds. J. A. Joines, R. R. Barton, K. Kang, and P. A. Fishwick, 449-445. Piscataway, New Jersey: Institute of Electrical and Electronics Engineers, Inc.

Kress, Reid, Dixon, Jack, Insalaco, Tom, and Rinehart, Richard, A Supply Chain Paradigm to Model Business Processes at the Y-12 National Security Complex, In Proceedings of the 2007 Winter Simulation Conference, eds. S. G. Henderson, B. Biller, M.-H Hsieh, J. Shortle, J. D. Tew, and R. R. Barton, 1977-1985. Piscataway, New Jersey: Institute of Electrical and Electronics Engineers, Inc.

Kress, Reid, Bills, Karen, Dixon, Jack, and Rinehart, Richard, Database-Intensive Process Simulation at the Y-12 National Security Complex, In Proceedings of the 2006 Winter Simulation Conference, eds. L. R. Perrone, F. P. Wieland, J. Liu, B. G. Lawson, D. M. Nicol, and R. M. Fujimoto, 1989-1996. Piscataway, New Jersey: Institute of Electrical and Electronics Engineers, Inc.

\section{AUTHOR BIOGRAPHIES}

REID KRESS is a Senior Technical Advisor in the National Security Technology Center at the National Nuclear
Security Administration's Y-12 Plant. He has a BS, MS, and $\mathrm{PhD}$ in Mechanical Engineering. He has worked in simulation modeling for the past decade. He is best reached via e-mail at $\langle$ kressrley12. doe.gov $>$.

KAREN BILLS received a B.S. degree in mechanical engineering and an M.S. degree in industrial engineering, in 1980 and 1993, respectively, both from the University of Tennessee, Knoxville. She has worked on simulations of hardware response to earthquakes, graphical simulation of mechanical designs, and robotic simulation for deactivation of the Department of Energy (DOE) Facilities. She may be reached via e-mail at $<$ billskc@y12.doe.gov $>$.

PETE BEREOLOS received his $\mathrm{PhD}$ in Chemical Engineering from Purdue University in 1995. He has an MS in Chemical Engineering from MIT and a BS in Chemical Engineering from Purdue University. He has been involved with the simulation modeling efforts at $\mathrm{Y}-12$ for the past two years. He can be reached via e-mail at $<$ bereolosp1@y12.doe.gov.

JAMES H. CLINTON received his PhD in Chemical Engineering from Purdue University. He has been involved in Extend/Modeling effort for several years. He recently concluded that scheduling was as important, or more important, than modeling. He may be reached through e-mail at <clintonjh@y12.doe.gov>.

JACK DIXON manages the simulation modeling efforts at $\mathrm{Y}-12$. His email address is $<$ dixonjr@y12.doe.gov $>$.

PHIL DUNN is retired from the Information Technology Division at the National Nuclear Security Agency's Y-12 Plant in Oak Ridge, TN. Prior to retirement, he was on the simulation modeling team at Y-12 for several years.

JULIE CHOATE MOORE is a Simulation Modeler in the Information Technology Division at the National Nuclear Security Agency's Y-12 Plant in Oak Ridge, TN. She has a BS degree in Mechanical Engineering from the University of Tennessee - Knoxville. Her e-mail address is $<$ moorejc@y12.doe.gov>.

ROBERT WILSON received a BS in Computer Science from VA Tech in 1984 and a MS in Industrial Engineering from the University of Tennessee in 1989. He specializes in the design, implementation and management of product lifecycle information systems in support of manufacturing. His e-mail address is wilsonrm2@y12.doe.gov. 